Pacific Journal of Mathematics

UNBOUNDED REPRESENTATIONS OF *-ALGEBRAS 


\section{UNBOUNDED REPRESENTATIONS OF *-ALGEBRAS}

\section{S. Gudder AND W. SCRUGGS}

Basic results on unbounded operator algebras are given, a general class of representations, called adjointable representations is introduced and irreducibility of representations is considered. A characterization of self-adjointness for closed, strongly cyclic *-representations is presented.

1. Introduction. Algebras of unbounded operators and unbounded representations of $*$-algebras have been important in quantum field theory $[1,3,9,10]$ and certain studies of Lie algebras $[5,7]$. The present paper proceeds along the lines initiated and developed by Robert Powers [6, 7] and much of the notation and definitions follow [6]. In \$2, we present some basic results concerning unbounded operator algebras, introduce a class of representations called adjointable representations, and consider irreducibility of representations. Section 3 characterizes the self-adjointness of closed, strongly cyclic *-representations.

2. Adjointable representations. Let $M$ and $N$ be subspaces (linear manifolds) in a Hilbert space $H$. Let $L(M, N)$ and $L_{c}(M, N)$ denote the collection of linear operators and closable linear operators, respectively with domain $M$ and range in $N$. For simplicity we use the notation $L(M)=L(M, M)$ and $L_{c}(M)=L_{c}(M, M)$. Notice that $L_{c}(H)$ is the set of bounded linear operators on $H$. We denote the domain of an operator $A$ by $D(A)$ and if $A$ is closable we denote the closure of $A$ by $\bar{A}$. A collection of operators $\mathscr{B}$ is an op-algebra if there exists a subspace $M$ such that $\mathscr{B} \subseteq L(M)$ and $A, B \in \mathscr{B}$ implies $A B,(\alpha A+B) \in \mathscr{B}$ for all $\alpha \in \mathbf{C}$. A set $\mathscr{B} \subseteq L(M)$ is symmetric if $M$ is dense and $A \in \mathscr{B}$ implies $D\left(A^{*}\right) \supseteq M$ and $A^{*} \mid M \in \mathscr{B}$. A symmetric op-algebra $\mathscr{B} \subseteq L(M)$ that contains $I \mid M$ is called an op ${ }^{*}$-algebra. It is easy to see that if $\mathscr{B} \subseteq L(M)$ is an op*-algebra, then the map $A \rightarrow A^{*} \mid M$ is an involution so $\mathscr{B}$ is a $*$-algebra. Also, if $\pi$ is a representation of a $*$-algebra $\mathscr{A}$, then $\pi(\mathscr{A})=\{\pi(A): A \in \mathscr{A}\}$ is an op-algebra and if $\pi$ is a $*$-representation of $\mathscr{A}$, then $\pi(\mathscr{A})$ is an $\mathrm{op}^{*}$-algebra (we always assume that a *-algebra contains an identity $I$ ).

A set $\mathscr{B} \subseteq L(M, N)$ is directed if for any $B_{1}, B_{2} \in \mathscr{B}$ there exists a $B_{3} \in \mathscr{B}$ such that $\left\|B_{1} x\right\|,\left\|B_{2} x\right\| \leqq\left\|B_{3} x\right\|$ for all $x \in M$. For example, if $\mathscr{B} \subseteq L_{c}(H)$ and $\{\lambda I: \lambda \geqq 0\} \subseteq \mathscr{B}$, then $\mathscr{B}$ is directed. Indeed, just let $B_{3}=\left(\left\|B_{1}\right\|+\left\|B_{2}\right\|\right) I$. For an example of an unbounded directed set, let $\mathscr{B} \subset L(M, H) \quad$ and suppose $B_{1}, B_{2} \in \mathscr{B}$ implies $B_{3}=$ 
$I \mid M+B_{1}^{*} B_{1}+B_{2}^{*} B_{2} \in \mathscr{B}$. Then for any $x \in M$ we have

$$
\begin{aligned}
\left\|B_{3} x\right\|^{2} & =\|x\|^{2}+\|\left(B_{1}^{*} B_{1}+B_{2}^{*} B_{2} x \|^{2}+2\left\langle\left(B_{1}^{*} B_{1}+B_{2}^{*} B_{2}\right) x, x\right\rangle\right. \\
& \geqq 2\left(\left\|B_{1} x\right\|^{2}+\left\|B_{2} x\right\|^{2}\right) \\
& \geqq\left\|B_{1} x\right\|^{2},\left\|B_{2} x\right\|^{2} .
\end{aligned}
$$

In particular, any op *-algebra is directed.

An extension $\mathscr{B}_{1}$ of $\mathscr{B} \subseteq L(M, N)$ is a set of operators $\mathscr{B}_{1} \subseteq$ $L\left(M_{1}, N_{1}\right)$ where $M \subseteq M_{1}, N \subseteq N_{1}$ and for which there exists a bijection $\phi: \mathscr{B} \rightarrow \mathscr{B}_{1}$ such that $\phi(B) \mid M=B$ for every $B \in \mathscr{B}$. If $\mathscr{B} \subseteq L(M, N)$, the $\mathscr{B}$-topology on $M$ is the topology generated by the set of seminorms $\{\|x\|,\|B x\|: B \in \mathscr{B}\}$. The completion of $M$ in the $\mathscr{B}$-topology is denoted by $\hat{M}_{\mathscr{B}}$ or simply $\hat{M}$ if no confusion can arise. We say that $\mathscr{B} \subseteq L(M, N)$ is collectively closed if for any net $x_{\alpha} \in M$ satisfying $x_{\alpha} \rightarrow x \in H, B x_{\alpha} \rightarrow y(B) \in H$ for every $B \in \mathscr{B}$, then $x \in M$ and $B x=$ $y(B)$. Clearly if all $B \in \mathscr{B}$ are closed then $\mathscr{B}$ is collectively closed; the converse need not hold.

THEOREM 1.

(1) $\mathscr{B} \subset L(M, N)$ is collectively closed if and only if $M=\hat{M}_{\mathscr{B}}$.

(2) If $\mathscr{B} \subseteq L_{c}(M, N)$, then the set $\mathscr{B}_{1}=\left\{\bar{B} \mid M_{1}: B \in \mathscr{B}\right\}$ where $M_{1}=$ $\cap\{D(\bar{B}): B \in \mathscr{B}\}$ is collectively closed.

(3) If $\mathscr{B} \subseteq L_{c}(M, N)$, then the set $\overline{\mathscr{B}}=\left\{\bar{B} \mid \hat{M}_{\mathscr{B}}: B \in \mathscr{B}\right\}$ is the minimal collectively closed extension of $\mathscr{B}$. Moreover, if $\mathscr{B} \subseteq L_{c}(M)$ and $A, B \in \mathscr{B}$ implies $A B \in \mathscr{B}$, then $\overline{\mathscr{B}} \subseteq L_{c}\left(\hat{M}_{\mathscr{B}}\right)$.

(4) If $\mathscr{B} \subseteq L_{c}(M, N)$ is directed, then $\hat{M}_{\mathscr{B}}=\cap\{D(\bar{B}): B \in \mathscr{B}\}$.

(5) If $\mathscr{B} \subseteq L_{c}(M)$ is an op-algebra, then $\overline{\mathscr{B}}$ is an op-algebra. If $\mathscr{B} \subseteq L_{c}(M)$ is an op *-algebra, then $\overline{\mathscr{B}}$ is an op *-algebra and $\hat{M}_{\mathscr{B}}=$ $\cap\{D(\bar{B}): B \in \mathscr{B}\}$.

\section{Proof.}

(1) Suppose $\mathscr{B} \subseteq L(M, N)$ is collectively closed and $x_{\alpha} \in M$ is a Cauchy net in the $\mathscr{B}$-topology. Then $x_{\alpha}$ and $B x_{\alpha}$ are Cauchy in $H$ so there exist $x, y(B) \in H$ such that $x_{\alpha} \rightarrow x, B x_{\alpha} \rightarrow y(B)$ in $H$ for every $B \in \mathscr{B}$. Since $\mathscr{B}$ is collectively closed, $x \in M$ and $B x_{\alpha} \rightarrow B x$, so $x_{\alpha} \rightarrow x$ in the $\mathscr{B}$-topology and $M$ is complete in the $\mathscr{B}$-topology. Hence $M=\hat{M}_{\mathscr{B}}$. Conversely, suppose $M=\hat{M}_{\mathscr{B}}$, and $x_{\alpha}$ is a net in $M$ such that $x_{\alpha} \rightarrow x$ and $B x_{\alpha} \rightarrow y(B)$ in $H$ for every $B \in \mathscr{B}$. Then $x_{\alpha}$ is Cauchy in the $\mathscr{B}$-topology. Since $M$ is complete in the $\mathscr{B}$-topology there exists an $x^{\prime} \in M$ such that $x_{\alpha} \rightarrow x^{\prime}$ and $B x_{\alpha} \rightarrow B x^{\prime}$ in $H$ for every $B \in \mathscr{B}$. Hence $x=x^{\prime} \in M$ and $B x=B x^{\prime}=y(B)$. 
(2) This is straightforward.

(3) It is clear that

$\hat{M}_{\mathscr{B}}=\left\{x \in \cap\{D(\bar{B}): B \in \mathscr{B}\}: M \ni x_{\alpha} \rightarrow x, \quad B x_{\alpha} \rightarrow \bar{B} x \quad\right.$ for $\quad$ all $B \in \mathscr{B}\}$. We now show that $\hat{M}_{\mathscr{B}}$ is complete in the $\overline{\mathscr{B}}_{\mid \hat{M}_{\mathscr{B}}}$ topology. Suppose $x_{\alpha} \in \hat{M}_{\mathscr{B}}$ is Cauchy in the $\overline{\mathscr{B}} \hat{M}_{\mathscr{B}}$-topology. Then $x_{\alpha}$ and $B x_{\alpha}$ are Cauchy in $H$ for every $B \in \mathscr{B}$. Hence there exists an $x \in H$ such that $x_{\alpha} \rightarrow x$ and $\bar{B} x_{\alpha} \rightarrow \bar{B} x$ for every $B \in \mathscr{B}$. Since $x_{\alpha} \in \hat{M}_{\mathscr{B}}$ there exists a net $x_{\alpha \beta} \in M$ such that $x_{\alpha \beta} \rightarrow x_{\alpha}$ and $B x_{\alpha \beta} \rightarrow \bar{B} x_{\alpha}$ in $H$ for every $B \in \mathscr{B}$. Now $x_{\alpha \beta}$ is a net in $M$ and $x_{\alpha \beta} \rightarrow x, B x_{\alpha \beta} \rightarrow \bar{B} x$ in $H$ for every $B \in \mathscr{B}$. Hence $x \in \hat{M}_{\mathscr{B}}$. It follows from (1) that $\overline{\mathscr{B}}$ is collectively closed. Clearly $\overline{\mathscr{B}}$ is an extension of $\mathscr{B}$. Moreover, $\overline{\mathscr{B}}$ is a minimal collectively closed extension since any collectively closed extension of $\mathscr{B}$ must contain $\hat{M}_{\mathscr{B}}$ in its domain. Now suppose $\mathscr{B} \subseteq L_{c}(M)$ and $A, B \in \mathscr{B}$. If $x \in \hat{M}_{\mathscr{B}}$, then there exists a net $x_{\alpha} \in M$ such that $x_{\alpha} \rightarrow x$ and $B x_{\alpha} \rightarrow \bar{B} x$ for every $B \in \mathscr{B}$. For fixed $A \in \mathscr{B}$ we have $A x_{\alpha} \in M$ and $A x_{\alpha} \rightarrow \bar{A} x$ and for every $B \in \mathscr{B}$, since $B A \in \mathscr{B}, B A x_{\alpha} \rightarrow \overline{B A} x=$ $\bar{B} \bar{A} x$. Hence $\bar{A} x \in \hat{M}_{\mathscr{B}}$ and $\overline{\mathscr{B}} \subseteq L_{c}\left(\hat{M}_{\mathscr{B}}\right)$.

(4) Suppose that $\mathscr{B} \subseteq L_{c}(M, N)$ is directed. We have seen that $\hat{M}_{\mathscr{B}} \subseteq \cap\{D(\bar{B}): B \in \mathscr{B}\}$. If $x \in \cap\{D(\bar{B}): B \in \mathscr{B}\}$, then for each $B \in$ $\mathscr{B}$ there exists a sequence $x(B, i) \in M$ such that $x(B, i) \rightarrow x$ and $B x(B, i) \rightarrow \bar{B} x$. For each $B \in \mathscr{B}$ and for each integer $n>0$ there exists an integer $n_{B}>0$ such that $\left\|x\left(B, n_{B}\right)-x\right\|<n^{-1}$ and $\left\|B x\left(B, n_{B}\right)-\bar{B} x\right\|<n^{-1}$. For $A, B \in \mathscr{B}$, define the order $\left(A, n_{A}\right)<$ $\left(B, m_{B}\right)$ if $\|A z\| \leqq\|B z\|$ for every $z \in M$ and $n<m$. Since $\mathscr{B}$ is directed, $\left\{\left(B, n_{B}\right)\right\}$ is a directed partially ordered set and $x\left(B, n_{B}\right)$ is a net. Notice that if $\|A z\| \leqq\|B z\|$ for every $z \in M$ then $\|\bar{A} y\| \leqq\|\bar{B} y\|$ for every $y \in \cap\{D(\bar{B}): B \in \mathscr{B}\}$. Indeed let $z_{\imath} \in M$ be a sequence such that $z_{i} \rightarrow y$ and $B z_{i} \rightarrow \bar{B} y$. Since $\left\|A z_{\imath}-A z_{j}\right\| \leqq\left\|B z_{i}-B z_{j}\right\|, A z_{i}$ is Cauchy and hence $A z_{i} \rightarrow \bar{A} y$. Therefore,

$$
\|\bar{A} y\|=\lim \left\|A z_{i}\right\| \leqq \lim \left\|B z_{i}\right\|=\|\bar{B} y\| .
$$

Clearly, $x\left(B, m_{B}\right) \rightarrow x$ and to show that $A x\left(B, m_{B}\right) \rightarrow \bar{A} x$ let $\epsilon>0$ and let $n>0$ be an integer such that $n^{-1}<\epsilon$. Then for $\left(B, m_{B}\right)>\left(A, n_{A}\right)$ we have

$$
\begin{aligned}
\left\|A x\left(B, m_{B}\right)-\bar{A} x\right\| & =\left\|\bar{A} x\left(B, m_{B}\right)-\bar{A} x\right\| \\
& \leqq\left\|\bar{B} x\left(B, m_{B}\right)-\bar{B} x\right\| \\
& <m^{-1}<n^{-1}<\epsilon .
\end{aligned}
$$

It follows that $x \in \hat{M}_{\mathscr{B}}$.

(5) This is a straightforward consequence of (2) and (3). 
In the work of $\mathrm{R}$. Powers [6] only hermitian representations are considered. But there are important representations that are not hermitian. For example, even if $\pi$ is hermitian, $\pi^{*}$ need not be. We therefore treat a larger class of representations, which we call adjointable, that includes $\pi^{*}$ whenever $\pi$ is hermitian.

Let $\mathscr{A}$ be a $*$-algebra and let $\pi, \pi_{1}$ be two representations of $\mathscr{A}$ with domains $D(\pi), D\left(\pi_{1}\right) \subseteq H$. We say that $\pi$ and $\pi_{1}$ are adjoint and write $\pi a \pi_{1}$, if $\langle\pi(A) x, y\rangle=\left\langle x, \pi_{1}\left(A^{*}\right) y\right\rangle$ for every $A \in \mathscr{A}$ and $x \in D(\pi)$, $y \in D\left(\pi_{1}\right)$. Notice that $a$ is a symmetric relation; that is $\pi a \pi_{1}$ if and only if $\pi_{1} a \pi$. Also, $\pi a \pi$ if and only if $\pi$ is hermitian. Furthermore, if $\pi a \pi_{1}$ and $\pi_{1} a \pi_{2}$ then $\pi(A)=\pi_{2}(A)$ on $D(\pi) \cap D\left(\pi_{2}\right)$ for every $A \in \mathscr{A}$ and if $D(\pi)=D\left(\pi_{2}\right)$ then $\pi=\pi_{2}$. We say that a representation $\pi$ is adjointable if there exists a representation $\pi_{1}$ such that $\pi a \pi_{1}$.

If $\pi$ is a representation of a $*$-algebra $\mathscr{A}$, we define $D\left(\pi^{*}\right)=$ $\cap\left\{D\left(\pi(A)^{*}\right): A \in \mathscr{A}\right\} \quad$ and $\pi^{*}(A)=\pi\left(A^{*}\right)^{*} \mid D\left(\pi^{*}\right) \quad$ for all $A \in \mathscr{A}$. (To save parentheses we use the notation $\pi(A)^{*}=$ $[\pi(A)]^{*}$.) In general, $\pi^{*}$ need not be a representation since, for one thing, $D\left(\pi^{*}\right)$ need not be dense. If $\pi$ is hermitian, then $\pi^{*}$ is a representation [6]. Hence, if $\pi$ is hermitian, then

$$
\langle\pi(A) x, y\rangle=\left\langle x, \pi(A)^{*} y\right\rangle=\left\langle x, \pi^{*}\left(A^{*}\right) y\right\rangle
$$

for every $A \in \mathscr{A}$ and $x \in D(\pi), y \in D\left(\pi^{*}\right)$ so $\pi a \pi^{*}$ and each is adjointable.

\section{THEOREM 2.}

(1) $\pi$ is adjointable if and only if $D\left(\pi^{*}\right)$ is dense.

(2) If $\pi$ is adjointable, then $\pi^{*}$ is a closed representation and is the largest representation adjoint to $\pi$.

(3) Suppose $\pi \subset \pi_{1}$. If $\pi_{1} a \pi_{2}$, then $\pi a \pi_{2}$. If $\pi_{1}$ is adjointable, then so is $\pi$ and $\pi_{1}{ }^{*} \subset \pi^{*}$.

(4) If $\pi$ is adjointable, then there exists a smallest closed representation $\bar{\pi}$ which extends $\pi$. If $\pi a \pi_{1}$, then $\bar{\pi} a \pi_{1}$.

(5) If $\pi$ is adjointable, then $\pi^{*}, \bar{\pi}$ are adjointable, $\pi^{* *}$ is a closed representation and $\pi \subset \bar{\pi} \subset \pi^{* *}, \pi^{* * *}=\pi^{*}, \bar{\pi}^{*}=\pi^{*}$.

(6) If $\pi$ is hermitian and $\pi_{1}$ is an hermitian extension of $\pi$, then $\pi \subset \pi_{1} \subset \pi^{*}$.

(7) If $\pi$ is hermitian, then $\pi^{* *}$ and $\bar{\pi}$ are hermitian and $\pi \subset \bar{\pi} \subset \pi^{* *} \subset \pi^{*}$.

Proof.

(1) If $\pi$ is adjointable and $\pi a \pi_{1}$ then $\pi_{1}\left(A^{*}\right) \subset \pi(A)^{*}$ for every $A \in \mathscr{A}$ so $D\left(\pi_{1}\right) \subseteq D\left(\pi^{*}\right)$ and $D\left(\pi^{*}\right)$ is dense. Conversely, suppose 
$D\left(\pi^{*}\right)$ is dense. For $x \in D(\pi), y \in D\left(\pi^{*}\right)$ we have

$$
\begin{aligned}
\left\langle\pi\left(A^{*}\right) x, \pi^{*}(B) y\right\rangle & =\left\langle\pi\left(A^{*}\right) x, \pi\left(B^{*}\right)^{*} y\right\rangle \\
& =\left\langle\pi\left(B^{*}\right) \pi\left(A^{*}\right) x, y\right\rangle \\
& =\left\langle\pi\left(B^{*} A^{*}\right) x, y\right\rangle \\
& =\left\langle x, \pi\left(B^{*} A^{*}\right)^{*} y\right\rangle .
\end{aligned}
$$

Hence $\pi^{*}(B) y \in D\left(\pi\left(A^{*}\right)^{*}\right)$ and $\pi\left(A^{*}\right)^{*} \pi^{*}(B) y=\pi\left(B^{*} A^{*}\right)^{*} y$ for every $A, B \in \mathscr{A}$. If follows that $\pi^{*}(B): D\left(\pi^{*}\right) \rightarrow D\left(\pi^{*}\right)$ and $\pi^{*}(A) \pi^{*}(B)=\pi\left((A B)^{*}\right)^{*}=\pi^{*}(A B)$. Moreover, $\pi^{*}$ is linear since for $x \in D(\pi), y \in D\left(\pi^{*}\right)$ we have

$$
\begin{aligned}
\left\langle\pi^{*}(\alpha A+B) y, x\right\rangle & =\left\langle\pi\left(\bar{\alpha} A^{*}+B^{*}\right)^{*} y, x\right\rangle \\
& =\left\langle y, \bar{\alpha} \pi\left(A^{*}\right) x\right\rangle+\left\langle y, \pi\left(B^{*}\right) x\right\rangle \\
& =\left\langle\left[\alpha \pi^{*}(A)+\pi^{*}(B)\right] y, x\right\rangle .
\end{aligned}
$$

It follows that $\pi^{*}$ is a representation and $\pi a \pi^{*}$.

(2) It was shown in (1) that $\pi^{*}$ is a representation if $\pi$ is adjointable. It follows from Theorem 1 (2) that $\pi^{*}$ is closed. If $\pi a \pi_{1}$ then $\langle\pi(A) x, y\rangle=\left\langle x, \pi_{1}\left(A^{*}\right) y\right\rangle$ for all $x \in D(\pi), y \in D\left(\pi_{1}\right)$. Hence, $D\left(\pi_{1}\right) \subseteq D\left(\pi^{*}\right)$ and $\pi_{1}\left(A^{*}\right) \subset \pi(A)^{*}=\pi^{*}\left(A^{*}\right)$ for every $A \in \mathscr{A}$ so $\pi_{1} \subset \pi^{*}$.

(3) Suppose $\pi \subset \pi_{1}$ and $\pi_{1} a \pi_{2}$. Then for every $x \in D(\pi), y \in$ $D\left(\pi_{2}\right)$ we have $\langle\pi(A) x, y\rangle=\left\langle\pi_{1}(A) x, y\right\rangle=\left\langle x, \pi_{2}\left(A^{*}\right) y\right\rangle$. Hence $\pi a \pi_{2}$. For all $x \in D(\pi), y \in D\left(\pi_{1}{ }^{*}\right)$ we have $\langle\pi(A) x, y\rangle=$ $\left\langle x, \pi_{1}{ }^{*}\left(A^{*}\right) y\right\rangle$. Hence $\pi a \pi_{1}{ }^{*}$ and by (2) we have $\pi_{1}{ }^{*} \subset \pi^{*}$.

(4) If $\pi$ is adjointable, then by (1), $D\left(\pi^{*}\right)$ is dense. Then $D\left(\pi(A)^{*}\right)$ is dense so $\pi(A)$ is closable for every $A \in \mathscr{A}$. Define $D(\bar{\pi})=\hat{D}(\pi)_{\mathscr{B}}$ where $\mathscr{B}=\{\pi(A): A \in \mathscr{A}\}$ and $\bar{\pi}(A)=\overline{\pi(A)} \mid D(\bar{\pi})$. It follows from Theorem 1 (3) that $\{\bar{\pi}(A): A \in \mathscr{A}\}$ is the minimal collectively closed extension of $\mathscr{B}$. It is straightforward to show that $\bar{\pi}$ is a representation and that $\pi a \pi_{1}$ implies $\bar{\pi} a \pi_{1}$.

(5) If $\pi$ is adjointable then so is $\pi^{*}$ and from (2) $\pi^{* *}$ is a closed representation. If $x \in D(\pi), y \in D\left(\pi^{*}\right)$ then for all $A \in \mathscr{A}$ we have $\left\langle\pi^{*}\left(A^{*}\right) y, x\right\rangle=\left\langle\pi(A)^{*} y, x\right\rangle=\langle y, \pi(A) x\rangle$. Hence $x \in \cap\left\{D\left[\pi^{*}\left(A^{*}\right)^{*}\right]: A \in \mathscr{A}\right\}=D\left(\pi^{* *}\right)$ and $\pi^{* *}(A) x=\pi^{*}\left(A^{*}\right)^{*} x=$ $\pi(A) x$ so $\pi \subset \pi^{* *}$. Since $\pi \subset \bar{\pi}$ we have by (3) that $\bar{\pi}^{*} \subset \pi^{*}$. Since $\pi a \pi^{*}$ from (4) we have $\bar{\pi} a \pi^{*}$. Hence by (2) $\pi^{*} \subset \bar{\pi}^{*}$ so $\pi^{*}=$ $\bar{\pi}^{*}$. By (3) $\pi^{* * *} \subset \pi^{*}$. Since $\pi^{*} a \pi^{* *}$, by (2) we have $\pi^{*} \subset \pi^{* * *}$ so $\pi^{* * *}=\pi^{*}$.

(6) For all $x \in D(\pi), y \in D\left(\pi_{1}\right), A \in \mathscr{A}$ we have 


$$
\langle\pi(A) x, y\rangle=\left\langle\pi_{1}(A) x, y\right\rangle=\left\langle x, \pi_{1}\left(A^{*}\right) y\right\rangle .
$$

Hence $\pi_{1} a \pi$ and by (2) $\pi_{1} \subset \pi^{*}$.

(7) It is shown in [6] that $\bar{\pi}$ is hermitian if $\pi$ is hermitian. Since $\pi$ is hermitian we have $\pi \subset \pi^{*}$. Applying (3) twice gives $\pi^{* *} \subset \pi^{* * *}$ so $\pi^{* *}$ is hermitian. Since $\pi^{* *}$ is closed we have from (2) that $\pi \subset \bar{\pi} \subset$ $\pi^{* *}$ and from (6) $\pi^{* *} \subset \pi^{*}$.

We now show that the extensions in (7) can be distinct. Let $\mathscr{A}$ be the free commutative *-algebra on one hermitian generator $A$. Define the representation $\pi$ of $\mathscr{A}$ on the Hilbert space $H=L^{2}[0,1]$ as follows: $D(\pi)=\left\{f \in C^{\infty}[0,1]: f^{(n)}(0)=f^{(n)}(1)=0, n=0,1,2, \ldots\right\} \pi(A)=-i d / d t$. It is straightforward to show that $\pi$ is hermitian and that $\pi=\bar{\pi}=$ $\pi^{* *} \varsubsetneqq \pi^{*}[\mathbf{8}]$. Now let $\pi_{1}$ be the representation of $\mathscr{A}$ on $H$ defined by:

$$
\begin{aligned}
& D\left(\pi_{1}\right)=\left\{f \in C^{\infty}[0,1]: f(0)=f(1), f^{(n)}(0)=f^{(n)}(1), n=1,2, \ldots\right\} \\
& \pi_{1}(A)=-i d / d t
\end{aligned}
$$

It is straightforward to show that $\pi_{1}$ is hermitian and that $\pi_{1}=\bar{\pi}_{1} \varsubsetneqq \pi_{1}{ }^{* *}=$ $\pi_{1}{ }^{*}[8]$.

We now consider commutants and irreducibility. If $\pi a \pi_{1}$, define $C\left(\pi, \pi_{1}\right)$ to be the set of operators $C \in L_{c}(H)$ satisfying $\langle C \pi(A) x, y\rangle=$ $\left\langle C x, \pi_{1}\left(A^{*}\right) y\right\rangle$ for every $x \in D(\pi), y \in D\left(\pi_{1}\right), A \in \mathscr{A}$. The proof of the following lemma is straightforward.

LEMMA 3.

(1) $C\left(\pi, \pi_{1}\right)$ is a weakly closed subspace of $L_{c}(H)$ containing $I$.

(2) $C\left(\pi, \pi_{1}\right)$

$$
=\left\{C \in L_{c}(H): C: D(\pi) \rightarrow D\left(\pi_{1}{ }^{*}\right), C \pi(A)=\pi_{1}{ }^{*}(A) C \mid D(\pi)\right\} .
$$

(3) $C \in C\left(\pi, \pi_{1}\right)$ if and only if $C^{*} \in C\left(\pi_{1}, \pi\right)$.

The commutant of a $*$-representation $\pi$ is defined as $\pi(\mathscr{A})^{\prime}=$ $C(\pi, \pi)$. It follows from Lemma 3 that $\pi(\mathscr{A})^{\prime}$ is a weakly closed, symmetric subspace of $L_{c}(H)$ containing $I$. However, $\pi(\mathscr{A})^{\prime}$ need not be a von Neumann algebra [6]. If $\pi$ is self-adjoint then $\pi(\mathscr{A})^{\prime}$ is a von Neumann algebra [6]. If $\pi$ is a *-representation, the strong commutant is defined by

$$
\pi(\mathscr{A})_{s}^{\prime}=\left\{C \in \pi(\mathscr{A})^{\prime}: C: D(\pi) \rightarrow D(\pi)\right\}
$$




$$
\begin{aligned}
\pi(\mathscr{A})_{s}^{\prime}= & \left\{C \in L_{c}(H): C: D(\pi) \rightarrow D(\pi), C \pi(A)\right. \\
& =\pi(A) C \mid D(\pi), \forall A \in \mathscr{A}\} .
\end{aligned}
$$

It is easy to see that $\pi(\mathscr{A})_{s}^{\prime}$ is an op-algebra in $L_{c}(H)$ containing $I$ and if $\pi$ is closed, then $\pi(\mathscr{A})_{s}^{\prime}$ is weakly closed [1]. Again $\pi(\mathscr{A})_{s}^{\prime}$ need not be a von Neumann algebra but if $\pi$ is self-adjoint, then $\pi(\mathscr{A})_{s}^{\prime}$ is a von Neumann algebra and $\pi(\mathscr{A})_{s}^{\prime}=\pi(\mathscr{A})^{\prime}$.

LEMMA 4. A *-representation $\pi$ is self-adjoint if and only if $\pi(\mathscr{A})^{\prime}=\pi(\mathscr{A})_{s}^{\prime}$ and $D\left(\pi^{*}\right)=\cup\left\{C x: x \in D(\pi), C \in \pi(\mathscr{A})^{\prime}\right\}$.

Proof. Necessity follows from our previous observations. For sufficiency, if $\pi(\mathscr{A})^{\prime}=\pi(\mathscr{A})_{s}^{\prime}$ then $C: D(\pi) \rightarrow D(\pi)$ for all $C \in$ $\pi(\mathscr{A})^{\prime}$. Hence $D\left(\pi^{*}\right)=\cup\left\{C x: x \in D(\pi), C \in \pi(\mathscr{A})^{\prime}\right\} \subset D(\pi)$.

For a bounded $*$-representation $\pi$ of a $*$-algebra $\mathscr{A}$ on a Hilbert space $H$ the following conditions are equivalent $[2,4]$.

(i) $\pi(\mathscr{A})^{\prime}=\{\lambda I: \lambda \in C\}$.

(ii) The only invariant closed subspaces of $H$ are $\{0\}$ and $H$.

(iii) Every nonzero vector in $H=D(\pi)$ is cyclic. A bounded *-representation $\pi$ is said to be irreducible if $\pi$ satisfies any one (and hence all) of these three conditions.

For unbounded self-adjoint representations one can give examples $[6,8]$ which show that no two of the above conditions are equivalent. Also, there is more than one natural way to extend some of the above conditions for unbounded self-adjoint representations. Let $\pi$ be a self-adjoint representation. We say that a subspace $M$ is a self-adjoint invariant subspace for $\pi$ if $M$ is invariant and $\pi \mid M$ is self-adjoint. The following are natural conditions that one might use to define irreducibility for a self-adjoint representation $\pi$ of a $*$-algebra $\mathscr{A}$ with domain $D(\pi) \subseteq H$.

(1) $\pi(\mathscr{A})^{\prime}=\{\lambda I: \lambda \in C\}$.

(2) The only invariant subspaces for $\pi$ which are complete in the $\pi(\mathscr{A})$-topology are $\{0\}$ and $D(\pi)$.

$\left(2^{\prime}\right)$ The only self-adjoint invariant subspaces for $\pi$ are $\{0\}$ and $D(\pi)$.

(3) Every nonzero vector in $D(\pi)$ is strongly cyclic.

$\left(3^{\prime}\right)$ Every nonzero vector in $D(\pi)$ is cyclic.

THEOREM 5. If $\pi$ is self-adjoint representation of the *-algebra $\mathscr{A}$ on the Hilbert space $H$, then 


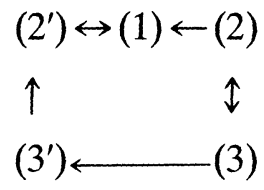

Proof. (2) $\rightarrow$ (3). Suppose (2) holds, $0 \neq \phi \in D(\pi)$ and $M=$ $\{\pi(A) \phi: A \in \mathscr{A}\}$. Clearly, $M \neq\{0\}$ and $M$ is an invariant subspace of $H$ for $\pi$. Let $\hat{M}$ be the completion of $M$ in the $\pi(\mathscr{A})$-topology. Since $\pi$ is closed, $\hat{M} \subseteq D(\pi)$ and clearly $\hat{M}$ is a subspace of $H$. We now show that $\hat{M}$ is invariant under $\pi$. If $x \in \hat{M}$, then there exists a net $x_{\alpha} \in M$ such that $x_{\alpha} \rightarrow x$ in the $\pi(\mathscr{A})$-topology. Fix an $A \in \mathscr{A}$. Then for every $B \in \mathscr{A}$ we have

$$
\pi(B) \pi(A) x_{\alpha}=\pi(B A) x_{\alpha} \rightarrow \pi(B A) x=\pi(B) \pi(A) x .
$$

Hence $\pi(A) x_{\alpha} \rightarrow \pi(A) x$ in the $\pi(\mathscr{A})$-topology so $\pi(A) x \in \hat{M}$ and $\pi(A) \hat{M} \subseteq \hat{M}$. Since (2) holds, $\hat{M}=D(\pi)$. Hence $M$ is dense in $D(\pi)$ in the $\pi(\mathscr{A})$-topology so $\phi$ is a strongly cyclic vector for $\pi$.

$(3) \rightarrow(2)$. Suppose (2) does not hold. Then there exists a $\pi(\mathscr{A})$ complete invariant subspace $M$ of $H$ with $M \neq\{0\}, D(\pi)$. If $0 \neq \phi \in M$, then clearly $\phi$ is not a strongly cyclic vector for $\pi$

$(1) \rightarrow\left(2^{\prime}\right)$. Suppose $\left(2^{\prime}\right)$ does not hold. Then there exists a nontrivial self-adjoint invariant subspace $M$ for $\pi$. Now $M$ is not dense in $H$ since otherwise $\pi \mid M$ is a $*$-representation of $\mathscr{A}$ on $\bar{M}=H$ with domain $M \subseteq D(\pi)$. Then $\pi \mid M \subset \pi=\pi^{*} \subset(\pi \mid M)^{*}$. Since $\pi \mid M$ is selfadjoint, $\pi \mid M=\pi$ and $D(\pi)=M$ which is a contradiction. By Theorem 4.7 [6] the projection $E$ on $\bar{M}$ satisfies $E \in \pi(\mathscr{A})^{\prime}$. Since $E \neq 0, I,(1)$ does not hold.

$\left(2^{\prime}\right) \rightarrow(1)$. Suppose (1) does not hold. Since $\pi$ is self-adjoint, $\pi(\mathscr{A})^{\prime}$ is a von Neumann algebra so there exists a nontrivial projection $E \in \pi(\mathscr{A})^{\prime}$. By Theorem $4.7[6], E D(\pi)$ is a nontrivial self-adjoint invariant subspace for $\pi$. Thus $\left(2^{\prime}\right)$ does not hold.

$\left(3^{\prime}\right) \rightarrow(1)$. Suppose $\left(3^{\prime}\right)$ holds. Let $0 \neq E \in \pi(\mathscr{A})^{\prime}$ be a projection. By Theorem $4.7[6], E D(\pi)=M$ is a self-adjoint invariant subspace for $\pi$. Let $0 \neq \phi \in M$. Since $\phi$ is cyclic and $\{\pi(A) \phi: A \in$ $\mathscr{A}\} \subseteq M, M$ is dense in $H$. As in (1) $\rightarrow\left(2^{\prime}\right)$ above, $M=D(\pi)$ and hence $E=I$. Since 0 and $I$ are the only projections in $\pi(\mathscr{A})^{\prime}$, we have $\pi(\mathscr{A})^{\prime}=\{\lambda I: \lambda \in C\}$.

$(3) \rightarrow\left(3^{\prime}\right)$. This is trivial. (2) $\rightarrow(1)$. Since $(2) \rightarrow\left(2^{\prime}\right)$ trivially, this follows from $\left(2^{\prime}\right) \rightarrow(1)$ above.

3. Closed strongly cyclic *-representations. In this section we shall mainly be concerned with characterizing self- 
adjointedness for closed strongly cyclic $*$-representations. Let $\pi$ be a *-representation of a *-algebra $\mathscr{A}$ with domain $D(\pi) \subseteq H$. The unbounded commutant $\pi(\mathscr{A})^{c}$ of $\pi$ is defined as the set of operators $C \in L(D(\pi), H)$ such that $\langle C \pi(A) x, y\rangle=\left\langle C x, \pi\left(A^{*}\right) y\right\rangle$ for all $x, y \in D(\pi)$ and $A \in \mathscr{A}$. The strong unbounded commutant is defined by $\quad \pi(\mathscr{A})_{s}^{c}=\left\{C \in \pi(\mathscr{A})^{c}: C D(\pi) \rightarrow D(\pi)\right\}$. Notice that $\pi(\mathscr{A})^{\prime} \mid D(\pi) \subset \pi(\mathscr{A})^{c}$ and $\pi(\mathscr{A})_{s}^{\prime} \mid D(\pi) \subseteq \pi(\mathscr{A})_{s}^{c}$. In fact,

$$
\begin{aligned}
& \pi(\mathscr{A})^{\prime}=\left\{\bar{C}: C \in \pi(\mathscr{A})^{c}, C \text { bounded }\right\} \\
& \pi(\mathscr{A})_{s}^{\prime}=\left\{\bar{C}: C \in \pi(\mathscr{A})_{s}^{c}, C \text { bounded }\right\}
\end{aligned}
$$

We say that a net $B_{\alpha} \in L(M, N)$ converges weakly to $B \in L(M, N)$ if $\left\langle B_{\alpha} x, y\right\rangle \rightarrow\langle B x, y\rangle$ for every $x, y \in M$. Moreover, $\mathscr{B} \subseteq L(M, N)$ is weakly closed if for any net $B_{\alpha} \in \mathscr{B}$ which converges weakly to some $B \in L(M, N)$ we have $B \in \mathscr{B}$. The proof of the next lemma is straightforward.

LEMMA 6.

(1) If $\pi$ is self-adjoint, then $\pi(\mathscr{A})^{c}=\pi(\mathscr{A})_{s}^{c}$.

(2) $\pi(\mathscr{A})^{c}=\left\{C \in L\left(D(\pi), D\left(\pi^{*}\right)\right): C \pi(A)=\pi^{*}(A) C, \forall A \in \mathscr{A}\right\}$.

(3) $\left.\pi(\mathscr{A})_{s}^{c}=C \in L(D(\pi)): C \pi(A)=\pi(A) C, \forall A \in \mathscr{A}\right\}$.

(4) $\pi(\mathscr{A})^{c}$ is a weakly closed subspace of $L\left(D(\pi), D\left(\pi^{*}\right)\right)$ containing $I \mid D(\pi)$.

(5) $\pi(\mathscr{A})_{s}^{c}$ is an op-algebra in $L(D(\pi))$.

(6) $\pi(\mathscr{A})^{c}=\pi(\mathscr{A})_{s}^{c}$ if and only if $\pi(\mathscr{A})^{c}$ is an op-algebra.

Let $\mathscr{A}$ be a $*$-algebra and let $\pi, \pi_{1}$ be $*$-representation of $\mathscr{A}$ on Hilbert spaces $H, H_{1}$, respectively. We say that $\pi$ and $\pi_{1}$ are equivalent, and write $\pi \cong \pi_{1}$, if there exists a unitary transformation $V$ from $H$ onto $H_{1}$ such that $V D(\pi)=D\left(\pi_{1}\right)$ and $\pi(A)=V^{*} \pi_{1}(A) V$ for every $A \in \mathscr{A}$.

Let $\omega$ be a state on $\mathscr{A}$. Then by the GNS construction for *-algebras [6], there exists a closed, strongly cyclic $*$-representation $\pi_{\omega}$ of $\mathscr{A}$ with strongly cyclic vector $x_{0}$ such that $\omega(A)=\left\langle\pi_{\omega}(A) x_{0}, x_{0}\right\rangle$ for every $A \in \mathscr{A}$. Moreover, if $\pi$ is any closed, strongly cyclic $*$-representation of $\mathscr{A}$ with strongly cyclic vector $y_{0}$ such that $\left\langle\pi(A) y_{0}, y_{0}\right\rangle=\omega(A)$ for every $A \in \mathscr{A}$ then $\pi \cong \pi_{\omega}[6]$.

We now characterize states $\omega$ such that $\pi_{\omega}$ is self-adjoint. A linear functional $F: \mathscr{A} \rightarrow C$ is $\omega$-bounded if for every $B \in \mathscr{A}$ there exists an $M_{B} \geqq 0$ such that $|F(B A)| \leqq M_{B} \omega\left(A^{*} A\right)^{1 / 2}$ for every $A \in \mathscr{A}$. For example, if $A_{\alpha} \in \mathscr{A}$ is a net such that $\omega\left(A_{\alpha}^{*} A^{*} A A_{\alpha}\right)$ is Cauchy for every $A \in \mathscr{A}$, then the functional $F(A)=\lim \omega\left(A_{\alpha}^{*} A\right)$ is $\omega$ bounded. Indeed, for every $B \in \mathscr{A}$ we have 


$$
\begin{aligned}
F(B A) \mid & =\lim \left|\omega\left(A_{\alpha}^{*} B A\right)\right|=\lim \left|\omega\left(A^{*} B^{*} A_{\alpha}\right)\right| \\
& \leqq \omega\left(A^{*} A\right)^{1 / 2} \lim \omega\left(A_{\alpha}^{*} B B^{*} A_{\alpha}\right)^{1 / 2} .
\end{aligned}
$$

If every $\omega$-bounded linear functional has the above form, then we call $\omega$ a Riesz state.

THEOREM 7. Let $\omega$ be a state on the *-algebra $\mathscr{A}$. Then $\pi_{\omega}$ is self-adjoint if and only if $\omega$ is a Riesz state.

Proof. Recall that $\pi_{\omega}$ is constructed as follows. Let $\mathscr{I}$ be the left ideal $\mathscr{I}=\left\{A \in \mathscr{A}: \omega\left(A^{*} A\right)=0\right\}$ and let $H_{0}$ be the inner product space consisting of equivalence classes $[A]$ in $\mathscr{A} / \mathscr{I}$ with inner product $\langle[A],[B]\rangle=\omega\left(B^{*} A\right)$. Let $H$ be the Hilbert space completion of $H_{0}$. Define a $*$-representation $\pi_{0}$ of $\mathscr{A}$ with domain $D\left(\pi_{0}\right)=H_{0}$ by $\pi_{0}(A)[B]=[A B]$. If $\pi_{\omega}=\bar{\pi}_{0}$, then $\pi_{\omega}$ is a closed, strongly cyclic *-representation with domain $D\left(\pi_{\omega}\right)=\hat{H}_{0 \pi_{0}(\mathscr{A})}$ and strongly cyclic vector [I]. Now suppose $\pi_{\omega}$ is self-adjoint and $F: \mathscr{A} \rightarrow C$ is $\omega$-bounded. If $\omega\left(A^{*} A\right)=0$, then $F(A)=0$ so $F: \mathscr{I} \rightarrow 0$. Hence $F$ can be considered as a linear functional on $H_{0}$. Since $|F([A])| \leqq M_{I}\|[A]\|, F$ is a continuous linear functional on $H_{0}$ and by the Riesz theorem there exists a $z \in H$ such that $F([A])=\langle[A], z\rangle$ for every $[A] \in H_{0}$. Now for every $B \in \mathscr{A}$ we have

$$
\begin{aligned}
\left\langle\pi_{0}(B)[A], z\right\rangle \mid & =|\langle[B A], z\rangle|=|F([B A])| \\
& =|F(B A)| \leqq M_{B}\|[A]\| .
\end{aligned}
$$

Hence $z \in D\left(\pi_{0}{ }^{*}\right)=D\left(\pi_{\omega}{ }^{*}\right)=D\left(\pi_{\omega}\right)$, so there exists a net $\left[A_{\alpha}\right] \in H_{0}$ which converges to $z$ in the $\pi_{0}(\mathscr{A})$-topology. Thus $\left[A A_{\alpha}\right]$ is Cauchy for every $A \in \mathscr{A}$. Finally, for every $A \in \mathscr{A}$ we have

$$
F(A)=\lim \left\langle[A],\left[A_{\alpha}\right]\right\rangle=\lim \omega\left(A_{\alpha}^{*} A\right) .
$$

Conversely, suppose $\omega$ is a Riesz state and $x \in D\left(\pi_{\omega}^{*}\right)$. Define the linear functional $F: \mathscr{A} \rightarrow C$ by $F(A)=\langle[A], x\rangle$. Then for every $A, B \in \mathscr{A}$ we have

$$
|F(B A)|=|\langle\pi(B)[A], x\rangle| \leqq M_{B}\|[A]\|=M_{B} \omega\left(A^{*} A\right)^{1 / 2}
$$

so $F$ is $\omega$-bounded. Hence there exists a net $A_{\alpha} \in \mathscr{A}$ such that $\omega\left(A_{\alpha}^{*} A^{*} A A_{\alpha}\right)$ is Cauchy for every $A \in \mathscr{A}$ and $F(A)=\lim \omega\left(A_{\alpha}^{*} A\right)$ for every $A \in \mathscr{A}$. It follows that $\left[A_{\alpha}\right]$ is Cauchy in the $\pi_{0}(\mathscr{A})$-topology and hence there exists a $y \in D\left(\pi_{\omega}\right)$ such that $\left[A_{\alpha}\right] \rightarrow y$. Furthermore, for every $A \in \mathscr{A}$ we have $F(A)=\lim \omega\left(A_{\alpha}^{*} A\right)=\lim \left\langle[A],\left[A_{\alpha}\right]\right\rangle=$ $\langle[A], y\rangle$. Hence $x=y \in D\left(\pi_{\omega}\right)$ and $\pi_{\omega}$ is self-adjoint. 
COROllary. A closed, strongly cyclic *-representation $\pi$ with strongly cyclic vector $x_{0}$ is self-adjoint if and only if the state $A \rightarrow\left\langle\pi(A) x_{0}, x_{0}\right\rangle$ is a Riesz state.

A state $\omega$ is faithful if $\omega\left(A^{*} A\right)=0$ implies $A=0$. A vector $x_{0} \in D(\pi)$ is separating if $\pi(A) x_{0}=0$ implies $\pi(A)=0$. If $\omega$ is faithful then the strongly cyclic vector $x_{0}$ for $\pi_{\omega}$ is separating. Conversely, if $x_{0}$ is separating, then $\omega\left(A^{*} A\right)=0$ implies $\pi_{\omega}(A)=0$. A representation $\pi$ of $\mathscr{A}$ is ultra-cyclic if there exists an $x_{0} \in D(\pi)$ such that $D(\pi)=$ $\left\{\pi(A) x_{0}: A \in \mathscr{A}\right\}$. We then call $x_{0}$ an ultra-cyclic vector. Ultra-cyclic representations are important because of the following result.

LEMMA 8. $\pi$ is a closed, strongly cyclic *-representation if and only if $\pi$ is the closure of an ultra-cyclic *-representation $\pi^{0}$.

Proof. Suppose $\pi$ is a closed, strongly cyclic $*$-representation of $\mathscr{A}$ with strongly cyclic vector $x_{0}$. Define $D\left(\pi^{0}\right)=\left\{\pi(A) x_{0}: A \in \mathscr{A}\right\}$ and $\pi^{0}(B) \pi(A) x_{0}=\pi(B A) x_{0}$. Then $\pi^{0}$ is an ultra-cyclic $*$-representation and $\bar{\pi}^{0}=\pi$. Conversely, if $\pi$ is the closure of an ultra-cyclic *representation $\pi^{0}$ with ultra-cyclic vector $x_{0}$, then $\pi$ is a closed *representation. Moreover, since $D(\pi)$ is the completion of $D\left(\pi^{0}\right)$ in the $\pi(\mathscr{A})$-topology, $x_{0}$ is a strongly cyclic vector for $\pi$.

We call $\pi^{0}$ in the proof of Lemma 8 the underlying ultra-cyclic *-representation for $\pi$. We can obtain information about $\pi$ by studying the simpler representation $\pi^{0}$. For example, a condition characterizing the essential self-adjointness of $\pi^{0}$ characterizes the self-adjointness of $\pi$. Moreover, $\pi^{0 *}=\pi^{*}$ and $\pi^{0}(\mathscr{A})^{\prime}=\pi(\mathscr{A})^{\prime}$.

Let $\pi$ be an arbitrary ultra-cyclic *-representation of $\mathscr{A}$ with a separating ultra-cyclic vector $x_{0}$. For $x \in D\left(\pi^{*}\right)$ define $\pi^{c}(x) \in$ $L\left(D(\pi), D\left(\pi^{*}\right)\right)$ by $\pi^{c}(x) \pi(A) x_{0}=\pi^{*}(A) x$. This is a well-defined operator since $\pi(A) x_{0}=\pi(B) x_{0}$ implies $\pi(A)=\pi(B)$. Then for every $y, z \in D(\pi)$ we have

$$
\left\langle\pi\left(A^{*}\right) y, z\right\rangle=\langle y, \pi(A) z\rangle=\langle y, \pi(B) z\rangle=\left\langle\pi\left(B^{*}\right) y, z\right\rangle .
$$

Hence $\pi\left(A^{*}\right)=\pi\left(B^{*}\right)$, so $\pi\left(A^{*}\right)^{*}=\pi\left(B^{*}\right)^{*}$ and finally

$$
\pi^{*}(A)=\pi\left(A^{*}\right)^{*}\left|D\left(\pi^{*}\right)=\pi\left(B^{*}\right)^{*}\right| D\left(\pi^{*}\right)=\pi^{*}(B)
$$

It is straightforward to see that $D(\pi)$ is a *-algebra with identity $x_{0}$ under the product $\left(\pi(A) x_{0}\right) \circ\left(\pi(B) x_{0}\right)=\pi(A B) x_{0}$ and involution $\left(\pi(A) x_{0}\right)^{*}=$ 
$\pi\left(A^{*}\right) x_{0}$. Moreover, for every $x, y, z \in D(\pi)$ we have $\langle x \circ y, z\rangle=$ $\left\langle y, x^{*} \circ z\right\rangle$.

THEOREM 9. Let $\pi$ be an ultra-cyclic *-representation of $\mathscr{A}$ with a separating, ultra-cyclic vector $x_{0}$.

(1) $\pi^{c}$ is a weakly continuous linear bijection from $D\left(\pi^{*}\right)$ into $\pi(\mathscr{A})^{c}$.

(2) The following statements are equivalent.

(a) $C x_{0} \in D(\pi)$ for every $C \in \pi(\mathscr{A})^{c}$.

(b) $\pi(\mathscr{A})^{c}$ is an op-algebra.

(c) $\pi$ is self-adjoint.

(3) $\pi(\mathscr{A})^{c}$ is an op *-algebra if and only if $\pi$ is self-adjoint and there exists an involution $b$ on the *-algebra $D(\pi)$ satisfying

$$
\left\langle x^{*}, y\right\rangle=\left\langle y^{b}, x\right\rangle
$$

for every $x, y \in D(\pi)$.

(4) If $\pi(\mathscr{A})^{c}$ is an op*-algebra, then $\pi^{c}$ is a weakly continuous ${ }^{b}$-anti-isomorphism of $D(\pi)$ onto $\pi(\mathscr{A})^{c}$.

Proof.

(1) Clearly, $\pi^{c}$ is linear. To show that $\pi^{c}$ maps $D\left(\pi^{*}\right)$ into $\pi(\mathscr{A})^{c}$, for $x \in D\left(\pi^{*}\right), A \in \mathscr{A}, z \in D(\pi)$ and $y=\pi(B) x_{0} \in D(\pi)$ we have

$$
\begin{aligned}
\left\langle\pi^{c}(x) \pi(A) y, z\right\rangle & =\left\langle\pi^{c}(x) \pi(A B) x_{0}, z\right\rangle \\
& =\left\langle\pi^{*}(A B) x, z\right\rangle=\left\langle\pi^{*}(B) x, \pi\left(A^{*}\right) z\right\rangle \\
& =\left\langle\pi^{c}(x) \pi(B) x_{0}, \pi\left(A^{*}\right) z\right\rangle=\left\langle\pi^{c}(x) y, \pi\left(A^{*}\right) z\right\rangle .
\end{aligned}
$$

To show that $\pi^{c}$ is surjective, let $C \in \pi(\mathscr{A})^{c}$. Then $C x_{0} \in D\left(\pi^{*}\right)$ and for any $y=\pi(A) x_{0} \in D(\pi)$ we have

$$
\begin{aligned}
\pi^{c}\left(C x_{0}\right) y & =\pi^{c}\left(C x_{0}\right) \pi(A) x_{0}=\pi^{*}(A) C x_{0} \\
& =C \pi(A) x_{0}=C y .
\end{aligned}
$$

To show that $\pi^{c}$ is injective, suppose that $x, x_{1} \in D\left(\pi^{*}\right)$ and $\pi^{c}(x)=$ $\pi^{c}\left(x_{1}\right)$. Then

$$
x=\pi^{*}(1) x=\pi^{c}(x) x_{0}=\pi^{c}\left(x_{1}\right) x_{0}=\pi^{*}(1) x_{1}=x_{1} .
$$

To show that $\pi^{c}$ is weakly continuous, suppose that $x_{i}, x \in D\left(\pi^{*}\right)$ and 
$x_{i} \rightarrow x$ in norm. Then for any $y=\pi(B) x_{0} \in D(\pi)$ and $z \in D(\pi)$ we have

$$
\begin{aligned}
\lim \left\langle\pi^{c}\left(x_{i}\right) y, z\right\rangle & =\lim \left\langle\pi^{*}(B) x_{i}, z\right\rangle \\
& =\lim \left\langle x_{i}, \pi\left(B^{*}\right) z\right\rangle=\left\langle x, \pi\left(B^{*}\right) z\right\rangle \\
& =\left\langle\pi^{*}(B) x, z\right\rangle=\left\langle\pi^{c}(x) \pi(B) x_{0}, z\right\rangle \\
& =\left\langle\pi^{c}(x) y, z\right\rangle .
\end{aligned}
$$

(2) (a) $\rightarrow$ (b). Suppose that (a) holds and $C \in \pi(\mathscr{A})^{c}, y=$ $\pi(A) x_{0} \in D(\pi)$. We then have $C y=C \pi(A) x_{0}=\pi^{*}(A) C x_{0}=$ $\pi(A) C x_{0} \in D(\pi)$. Hence, by Lemma $6(6), \pi(\mathscr{A})^{c}$ is an op-algebra.

(b) $\rightarrow$ (c). If $x \in D\left(\pi^{*}\right)$, then by (1) $\pi^{c}(x) \in \pi(\mathscr{A})^{c}$. If $\pi(\mathscr{A})^{c}$ is an op-algebra, then $x=\pi^{*}(1) x=\pi^{c}(x) x_{0} \in D(\pi)$. Hence $D\left(\pi^{*}\right)=$ $D(\pi)$ and $\pi$ is self-adjoint.

(c) $\rightarrow$ (a). If $\pi$ is self-adjoint, then $\pi(\mathscr{A})^{c} \subseteq L(D(\pi))$.

(3) Suppose $\pi(\mathscr{A})^{c}$ is an op*-algebra. Then, by (2), $\pi$ is selfadjoint. If $C \in \pi(\mathscr{A})^{c}$, then $D(\pi) \subseteq D\left(C^{*}\right)$ and $C^{*} \mid D(\pi) \in \pi(\mathscr{A})^{c}$ so $C^{*}: D(\pi) \rightarrow D(\pi)$. For $x \in D(\pi)$, by (1) $\pi^{c}(x) \in \pi(\mathscr{A})^{c}$ so $x^{b} \equiv$ $\pi^{c}(x)^{*} x_{0} \in D(\pi)$. For $x=\pi(A) x_{0} \in D(\pi)$ and $y \in D(\pi)$ we have

$$
\begin{aligned}
\left\langle y^{b}, x\right\rangle & =\left\langle\pi^{c}(y)^{*} x_{0}, x\right\rangle=\left\langle x_{0}, \pi^{c}(y) \pi(A) x_{0}\right\rangle \\
& =\left\langle x_{0}, \pi(A) y\right\rangle=\left\langle\pi\left(A^{*}\right) x_{0}, y\right\rangle=\left\langle x^{*}, y\right\rangle
\end{aligned}
$$

so (3.1) holds. That ${ }^{b}$ is an involution now follows from (3.1). For example,

$$
\begin{aligned}
\left\langle(y \circ z)^{b}, x\right\rangle & =\left\langle x^{*}, y \circ z\right\rangle=\left\langle y^{*} \circ x^{*}, z\right\rangle \\
& =\left\langle z^{b}, x \circ y\right\rangle=\left\langle x^{*} \circ z^{b}, y\right\rangle=\left\langle y^{b}, z^{\left.b^{*} \circ x\right\rangle}\right. \\
& =\left\langle z^{b} \circ y^{b}, x\right\rangle .
\end{aligned}
$$

The other properties of an involution follow in a similar way. Conversely, suppose $\pi$ is self-adjoint and there exists an involution $^{b}$ on $D(\pi)$ satisfying (3.1). Then by (2), $\pi(\mathscr{A})^{c}$ is an op-algebra. If $C \in \pi(\mathscr{A})^{c}$, then for any $x=\pi(B) x_{0} \in D(\pi)$ and $y=\pi(A) x_{0} \in D(\pi)$ we have

$$
\begin{aligned}
\langle C y, x\rangle & =\left\langle C \pi(A) x_{0}, x\right\rangle=\left\langle C x_{0}, \pi\left(A^{*}\right) x\right\rangle \\
& =\left\langle C x_{0}, \pi\left(A^{*} B\right) x_{0}\right\rangle=\left\langle C x_{0},\left[\pi\left(B^{*} A\right) x_{0}\right]^{*}\right\rangle \\
& =\left\langle\pi\left(B^{*} A\right) x_{0},\left(C x_{0}\right)^{b}\right\rangle=\left\langle\pi(A) x_{0}, \pi(B)\left[\left(C x_{0}\right)^{b}\right]\right\rangle \\
& =\left\langle y, \pi^{c}\left[\left(C x_{0}\right)^{b}\right] x\right\rangle .
\end{aligned}
$$

Hence $D(\pi) \subseteq D\left(C^{*}\right), C^{*} \mid D(\pi)=\pi^{c}\left[\left(C x_{0}\right)^{b}\right] \in \pi(\mathscr{A})^{c}$ and so $\pi(\mathscr{A})^{c}$ is an op*-algebra. 
(4) Suppose $\pi(\mathscr{A})^{c}$ is an op*-algebra. It follows from (1) that $\pi^{c}: D(\pi) \rightarrow \pi(\mathscr{A})^{c}$ is a weakly continuous linear bijection. For $x=$ $\pi(A) x_{0} \in D(\pi)$ and $y=\pi(B) x_{0} \in D(\pi)$ we have

$$
\pi^{c}(x) y=\pi^{c}(x) \pi(B) x_{0}=\pi(B) x=\pi(B) x_{0} \circ \pi(A) x_{0}=y \circ x .
$$

It is now clear that $\pi^{c}$ is an anti-isomorphism. To show that $\pi^{c}$ is a ${ }^{b}$ anti-isomorphism, for $x \in D(\pi)$ and $y=\pi(A) x_{0} \in D(\pi)$ we have

$$
\begin{aligned}
\pi^{c}\left(x^{b}\right) y & =y \circ x^{b}=y \circ\left[\pi^{c}(x)^{*} x_{0}\right]=\pi(A) \pi^{c}\left(x^{*}\right) x_{0} \\
& =\pi^{c}(x)^{*} \pi(A) x_{0}=\pi^{c}(x)^{*} y .
\end{aligned}
$$

COROllary. Let $\pi$ be a closed, strongly cyclic $*$-representation of $\mathscr{A}$ with separating, strongly cyclic vector $x_{0}$ and let $\pi^{0}$ be the underlying ultra-cyclic representation. Then $\pi$ is self-adjoint if and only if $C x_{0} \in$ $D(\pi)$ for every $C \in \pi^{0}(\mathscr{A})^{c}$.

Proof. If $\pi$ is self-adjoint and $C \in \pi^{0}(\mathscr{A})^{c}$, then $C x_{0} \in D\left(\pi^{0 *}\right)=$ $D\left(\pi^{*}\right)=D(\pi)$. Conversely, suppose $C x_{0} \in D(\pi)$ for every $C \in$ $\pi^{0}(\mathscr{A})^{c}$. If $x \in D\left(\pi^{*}\right)$, then $x \in D\left(\pi^{0 *}\right)$ so by Theorem $9(1), \pi^{c}(x) \in$ $\pi^{0}(\mathscr{A})^{c}$. Hence $x=\pi^{c}(x) x_{0} \in D(\pi)$, so $D(\pi)=D\left(\pi^{*}\right)$ and $\pi$ is selfadjoint.

\section{REFERENCES}

1. H.J. Borchers and J. Yngvason, On the algebra of field operators. The weak commutant and integral decomposition of states, Commun. Math. Phys. 42 (1975), 231-252.

2. G.G. Emch, Algebraic Methods in Statistical Mechanics and Quantum Field Theory, John Wiley \& Sons, Inc., New York (1972).

3. G. Lassner, Topological algebras of operators, Rep. Math. Phys., 3 (1972), 279-293.

4. M.A. Naimark, Normed Rings, Noordhoff Publishers, Groningen, The Netherlands (1970).

5. E. Nelson, Analytic vectors, Ann. Math., 70 (1959), 572-615.

6. R.T. Powers, Self-adjoint algebras of unbounded operators, Commun. Math. Phys., 21 (1971), 85-124.

7. - Self-adjoint algebras of unbounded operators II, Trans. Amer. Math. Soc., 187 (1974), $1-33$.

8. W.M. Scruggs, Unbounded representations of *-algebras, Dissertation, University of Denver (1976).

9. B. Simon, The $P(\phi)_{2}$ Euclidean (Quantum) Field Theory, Princeton University Press, Princeton, New Jersey (1974).

10. R.F. Streater and A.S. Wightman, PCT, Spin and Statistics and All That, W.A. Benjamin, Inc., New York (1964).

Received January 13, 1977 and in revised form May 19, 1977. 


\section{PACIFIC JOURNAL OF MATHEMATICS \\ EDITORS}

RICHARD ARENS (Managing Editor)

University of California

Los Angeles, CA 90024

\section{R. A. Beaumont \\ University of Washington \\ Seattle, WA 98105}

C. C. MOORE

University of California

Berkeley, CA 94720

\section{J. DUGUNDJI}

Department of Mathematics

University of Southern California

Los Angeles, CA 90007

R. FINN AND J. MILGRAM

Stanford University

Stanford, CA 94305

\section{ASSOCIATE EDITORS}

\section{E. F. BECKENBACH}

B. H. NeUmanN

F. WOLF

K. YoSHIDA

\section{SUPPORTING INSTITUTIONS}

\author{
UNIVERSITY OF BRITISH COLUMBIA \\ CALIFORNIA INSTITUTE OF TECHNOLOGY \\ UNIVERSITY OF CALIFORNIA \\ MONTANA STATE UNIVERSITY \\ UNIVERSITY OF NEVADA \\ NEW MEXICO STATE UNIVERSITY \\ OREGON STATE UNIVERSITY \\ UNIVERSITY OF OREGON \\ OSAKA UNIVERSITY
}

\author{
UNIVERSITY OF SOUTHERN CALIFORNIA \\ STANFORD UNIVERSITY \\ UNIVERSITY OF HAWAII \\ UNIVERSITY OF TOKYO \\ UNIVERSITY OF UTAH \\ WASHINGTON STATE UNIVERSITY \\ UNIVERSITY OF WASHINGTON \\ AMERICAN MATHEMATICAL SOCIETY
}

The Supporting Institutions listed above contribute to the cost of publication of this Journal, but they are not owners or publishers and have no responsibility for its contents or policies.

Mathematical papers intended for publication in the Pacific Journal of Mathematics should be in typed form or offset-reproduced (not dittoed), double spaced with large margins. Underline Greek letters in red, German in green, and script in blue. The first paragraph or two must be capable of being used separately as a synopsis of the entire paper. Items of the bibliography should not be cited there unless absolutely necessary, in which case they must be identified by author and Journal, rather than by item number. Manuscripts, in duplicate, may be sent to any one of the four editors. Please classify according to the scheme of Math. Reviews, Index to Vol. 39. All other communications should be addressed to the managing editor, or Elaine Barth, University of California, Los Angeles, California, 90024.

100 reprints are provided free for each article, only if page charges have been substantially paid. Additional copies may be obtained at cost in multiples of 50 .

The Pacific Journal of Mathematics is issued monthly as of January 1966. Regular subscription rate: $\$ 72.00$ a year (6 Vols., 12 issues). Special rate: $\$ 36.00$ a year to individual members of supporting institutions.

Subscriptions, orders for back numbers, and changes of address should be sent to Pacific Journal of Mathematics, 103 Highland Boulevard, Berkeley, California, 94708.

PUBLISHED BY PACIFIC JOURNAL OF MATHEMATICS, A NON-PROFIT CORPORATION

Printed at Jerusalem Academic Press, POB 2390, Jerusalem, Israel.

Copyright (C) 1977 Pacific Journal of Mathematics All Rights Reserved 


\section{Pacific Journal of Mathematics \\ Vol. 70, No. $2 \quad$ October, 1977}

B. Arazi, A generalization of the Chinese remainder theorem ........... 289

Thomas E. Armstrong, Polyhedrality of infinite dimensional cubes .... . . . 297

Yoav Benyamini, Mary Ellen Rudin and Michael L. Wage, Continuous

images of weakly compact subsets of Banach spaces ............ 309

John Thomas Burns, Curvature functions on Lorentz 2-manifolds ......... 325

Dennis F. De Riggi and Nelson Groh Markley, Shear distality and equicontinuity .................................. 337

Claes Fernström, Rational approximation and the growth of analytic

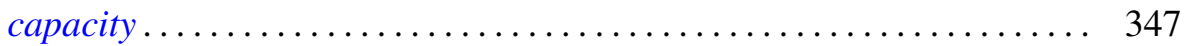

Pál Fischer, On some new generalizations of Shannon's inequality....... 351

Che-Kao Fong, Quasi-affine transforms of subnormal operators ......... 361

Stanley P. Gudder and W. Scruggs, Unbounded representations of

*-algebras........................................ 369

Chen F. King, A note on Drazin inverses .................... 383

Ronald Fred Levy, Countable spaces without points of first countability . . . 391

Eva Lowen-Colebunders, Completeness properties for convergence

spaces ......................................... 401

Calvin Cooper Moore, Square integrable primary representations ....... 413

Stanisław G. Mrówka and Jung-Hsien Tsai, On preservation of

E-compactness ................................ 429

Yoshiomi Nakagami, Essential spectrum $\Gamma(\beta)$ of a dual action on a von

Neumann algebra ................................ 437

L. Alayne Parson, Normal congruence subgroups of the Hecke groups

$G\left(2^{(1 / 2)}\right)$ and $G\left(3^{(1 / 2)}\right)$...

Louis Jackson Ratliff, Jr., On the prime divisors of zero in form rings . . . . 489

Caroline Series, Ergodic actions of product groups .................. 519

Robert O. Stanton, Infinite decomposition bases..................... 549

David A. Stegenga, Sums of invariant subspaces .................. 567 